\title{
Polarimetric surface plasmon resonance imaging biosensor
}

\author{
Aurélien Duval, ${ }^{1, *}$ Aude Laisné, ${ }^{2}$ Denis Pompon, ${ }^{2}$ Sylvain Held, ${ }^{3}$ Alain Bellemain, ${ }^{1}$ \\ Julien Moreau, ${ }^{1}$ and Michael Canva ${ }^{1}$ \\ ${ }^{1}$ Laboratoire Charles Fabry de l'Institut d'Optique, CNRS, Université Paris Sud, Campus Polytechnique RD 128, \\ 91127 Palaiseau CEDEX, France \\ ${ }^{2}$ Centre de Génétique Moléculaire, CNRS, Avenue de la Terrasse, 91190 Gif-sur-Yvette, France \\ ${ }^{3}$ Institut d'Electronique Fondamentale, CNRS, Université Paris Sud, Bâtiment 220, 91405 Orsay, France \\ *Corresponding author: aurelien.duval@institutoptique.fr
}

Received September 28, 2009; accepted October 13, 2009; posted November 2, 2009 (Doc. ID 117883); published November 19, 2009

We report the realization of a polarimetric surface plasmon resonance imaging system capable of dynamically resolving a change in the optical anisotropy of biochemical films. Anisotropies as small as $10^{-3}$ refractive index unit on nanometer-thick samples can be resolved. As an example, we present here the dynamical anisotropy obtained by the electrical patterning of a film consisting of a self-assembled monolayer deposited on gold, covered with a phospholipid hemimembrane. (C) 2009 Optical Society of America

OCIS codes: $240.6680,310.6860,110.5405$.

Surface plasmon resonance (SPR) biosensors are being increasingly used in laboratories as sensitive biochemical transducers for several applications such as genetic diagnostics [1], kinetics of interactions analysis [2], or food analysis [3], for example. They rely on the coupling between oscillating bound-free electrons present in a metallic thin-film (the surface plasmons) and an incident TM-polarized light wave. A modification in the refractive index of the surrounding dielectric medium will change the coupling between the plasmon waves and the incident light. Monitoring the reflectivity of such a system enables the detection of minute changes in the refractive index of the surrounding medium. Surface plasmon resonance imaging (SPRI) allows parallel monitoring of multiple interactions dynamically [4].

The polarimetric surface plasmon resonance imaging (P-SPRI) system demonstrated here is based on the Kretschmann-Raether configuration [5], using two orthogonal SPRI arms both capable of capturing TM-polarized images of the biosensor's surface, following two orthogonal planes of incidence, as shown in Fig. 1. Each sensor arm can act as a completely independent SPRI system. Compared to approaches based on coupled plasmon-waveguide resonance spectroscopy [6], or more classical ellipsometric methods [7], the P-SPRI system developed is capable of dynamical measurements on label-free films.

The reflectivity variations in the image measured in each direction ( $x$ and $y$ ) of the surface of the biosensor, shown in Eq. (1), are proportional to the variations in the effective refractive indices $\left(\Delta n_{x}\right.$ and $\Delta n_{y}$ ) probed by the propagating plasmon wave, as well as the thickness $(\Delta d)$ of the biological thin-film (typically 1-50 $\mathrm{nm}$ thick) deposited on the biosensor surface,

$$
\Delta R_{k} \propto \Delta n_{k} \Delta d, \quad k=\{x, y\} .
$$

Variations in the relative mean anisotropy $\Delta n$ of the covering dielectric medium are measured by subtracting the signal of one arm from the other. In bio- chemical systems consisting of long chains, like the self-assembled monolayer (SAM), the birefringence is given by the intrinsic anisotropy of the molecule and the order parameter of the assembly. The latter can be characterized by its azimuth $\phi$ and zenith $\theta$. An (arbitrary) 1-nm-thick equivalent layer of dense birefringent rod-shaped molecules with an intrinsic anisotropy of $\approx 0.1$ ( $n_{\text {iso }} \approx 1.45$ [8]) tethered to gold and oriented parallel to the surface $\left(\theta=90^{\circ}\right)$, and one of the system's axes $\left(\phi=0^{\circ}\right)$ will yield a maximal relative mean effective anisotropy of $\Delta n \approx \pm 0.1$ as measured by our system. Taking for reference a layer oriented along the surface normal $\left(\theta=0^{\circ}\right)$, the rotation of the molecules from "up" to "laying flat" will give rise to a simulated maximal reflectivity difference $\Delta R_{\text {aniso }}=\Delta R_{x}-\Delta R_{y}$ of about $\pm 3 \%$. In real applications, the signal measured will be reduced owing to smaller $\theta$ variations and an arbitrary azimuth.

For each orthogonal sensor arm, the incident light (an LED at $670 \mathrm{~nm}$, FWHM of $25 \mathrm{~nm}$ ) is first collimated, TM-polarized, and reflected off a motorized rotating mirror before being coupled to the metallic

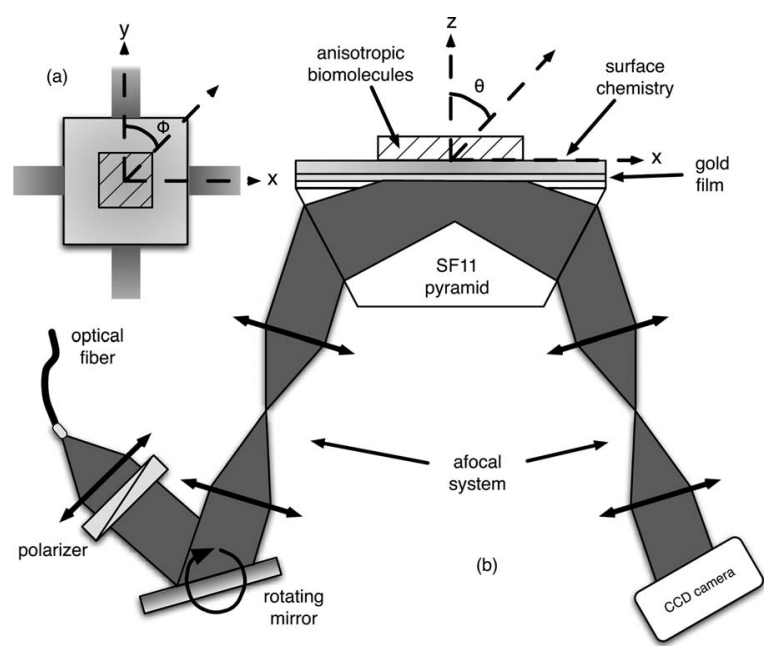

Fig. 1. Schematic representation of the P-SPRI system. (a) Top view. (b) Side view. 
sensor layer through the use of an afocal system and the SF11 pyramid. On the opposite face of the pyramid, the reflected light passes through a similar afocal system and is collected by a CCD camera (Cooke Pixelfly QE). Using an afocal optical system (magnification of $1 \times$ ) and a rotating mirror limits the angles of incidence to $\mathrm{a} \pm 5^{\circ}$ region around the central angle $\left(53^{\circ}\right.$ with respect to the base of the pyramid), hence limiting the range of measurable bulk refractive indices from 1.294 to 1.386. For dielectric layers ( $n$ $=1.45)$ deposited on gold and immersed in water $(n$ $=1.33$ ), it restricts the measurements to thin films $(<50 \mathrm{~nm})$.

The combined reading of both arms is shot-noise limited as in more traditional SPRI systems. We determined that our P-SPRI setup yields a noise of less than $10^{-2} \%$ using spatial averaging $(400 \mu \mathrm{m}$ $\times 400 \mu \mathrm{m}$ ), while keeping dynamical capabilities (1 s integration for 16 images averaged).

The anisotropy figure measured for each pixel of the resulting differential SPR images can only make sense if the P-SPRI system is calibrated so that isotropic samples do not appear as anisotropic. To demonstrate the normalization technique used, we carried out an experiment using a SF11 glass-slide covered with gold squares $\left(1 \mathrm{~mm}^{2}\right)$. The squares were deposited using lift-off UV lithography and vacuum evaporation techniques. They are composed of a 7-nm-thick Ti adhesion layer and a 47-nm-thick Au sensing layer. Index-matching oil was deposited between the base of the pyramid and the biosensor. The system was finally covered with a flow-cell.

At a fixed incidence angle (the maximum slope of the angular reflectivity curve), a dynamical SPRI measurement was performed simultaneously on nine gold squares (using $400 \mu \mathrm{m} \times 400 \mu \mathrm{m}$ spatial averaging) under a constant flow of water. After approximately $2 \mathrm{~min}$, water was replaced with a mixture of $1 \%$ volume per volume (v/v) glycerol in water. After 6 min, water was injected and circulated until reaching a steady state. A second mixture of $1.5 \%(\mathrm{v} / \mathrm{v})$ glycerol was injected after approximately $10 \mathrm{~min}$.

Figures 2(a) and 2(b) show the reflectivity variations $\left(\Delta R_{x}\right.$ and $\left.\Delta R_{y}\right)$ induced by the injection of glycerol, averaged over nine gold squares. They correspond to bulk refractive index increases of, respectively, $\Delta n=1.4 \times 10^{-3}$ refractive index unit (RIU) for $1 \%$ glycerol and $\Delta n=2.1 \times 10^{-3}$ RIU for $1.5 \%$ glycerol. The resulting signal is equivalent to a thinfilm deposition as in biosensing applications. Using the extended Rouard method [9], we determined that the response to $1 \%$ glycerol would give a signal similar to the deposition of about $2 \mathrm{~nm}$ of a dielectric material $(n=1.45)$ on the gold surface.

To account for small differences in reflectivity slopes between the two arms, reflectivity variations are normalized by the mean $1 \%$ glycerol plateau. The dynamical SPR anisotropy signal $\left(\Delta R_{\text {aniso }}\right)$ can then be determined by subtracting arms $x$ and $y$ signals. The result for our glycerol experiment is depicted in Fig. 2(c). As expected, no artifactual anisotropy is found outside the injection and washing regions (materialized by dashed lines in the figure). In this
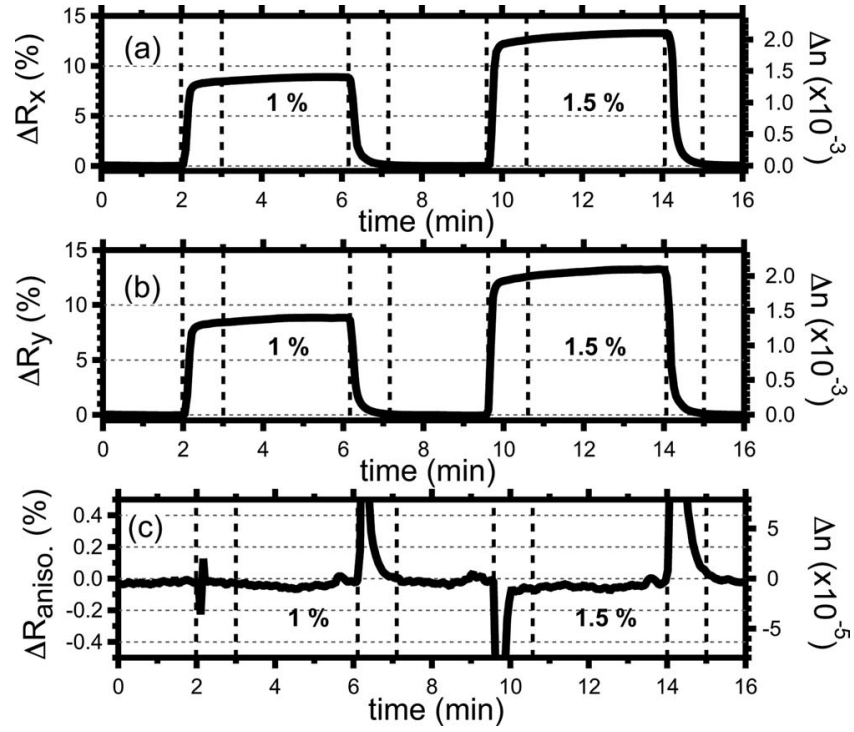

Fig. 2. Corrected mean dynamical reflectivity variations induced by the injections of $1 \%$ and $1.5 \%$ glycerol in water. (a) Arm $x$. (b) Arm $x$. (c) "Anisotropy" of the bulk liquid. Dashed lines represent fluidic transitions. Right axes, corresponding bulk $\Delta n$.

particular experiment, the residual noise is measured below $\Delta R_{\text {aniso }}=10^{-2} \%$, which would translate into a minimal detectable bulk anisotropy of $\Delta n_{\min }$ $=10^{-5} \mathrm{RIU}$ or into a minimal anisotropy of $10^{-3} \mathrm{RIU}$ for $1 \mathrm{~nm}$ of biomolecules $\left(n_{\text {iso }}=1.45\right)$ tethered onto the gold surface. Using our model of a dense layer of birefringent rod-shaped molecules with an intrinsic anisotropy of $\approx 0.1$, angular variations as small as $5^{\circ}$ of a 1-nm-thick equivalent layer can be discriminated.

To illustrate applications of such a system, the anisotropy resulting from the patterning of a surface consisting in a SAM and a phospholipid hemimembrane was determined dynamically using our P-SPRI setup. The biochip was divided into three different regions depicted in Fig. 3(b). Regions A and B were connected to a potentiostat capable of applying an electrical potential difference between these electrodes and the buffer solution. The REF region was acting as a reference for the electric cycles, and region $\mathrm{C}$ was used as a control and thus isolated from the surrounding electrodes.

Prior to the experiment, a layer of alkanethiols was allowed to self-assemble on the gold surface, covering all regions of the biochip. A fusion of phospholipid liposomes was then realized on the SAM, resulting in a supported hemimembrane [10]. During the experiment, several electrical cycles were realized between the $\mathrm{A}$ and $\mathrm{B}$ regions. Each cycle consisted into a $1 \mathrm{~min}$ square profile, applying a potential of $\pm 1.5 \mathrm{~V}$ to the region. The mean dynamical SPR anisotropy $\left(\Delta R_{\text {aniso }}\right)$ signal of the regions was captured using the P-SPRI system and is given in Fig. 3(a), showing parallel measurements on regions $\mathrm{A}$ (dots) and $\mathrm{C}$ (line). Spatial averaging (area of $\approx 400 \mu \mathrm{m} \times 400 \mu \mathrm{m}$ ) was used again in order to reduce the noise, and the signal resulting from the REF region was subtracted from the curves.

It can be clearly observed that region $\mathrm{C}$ stays isotropic throughout the experiment with a residual av- 


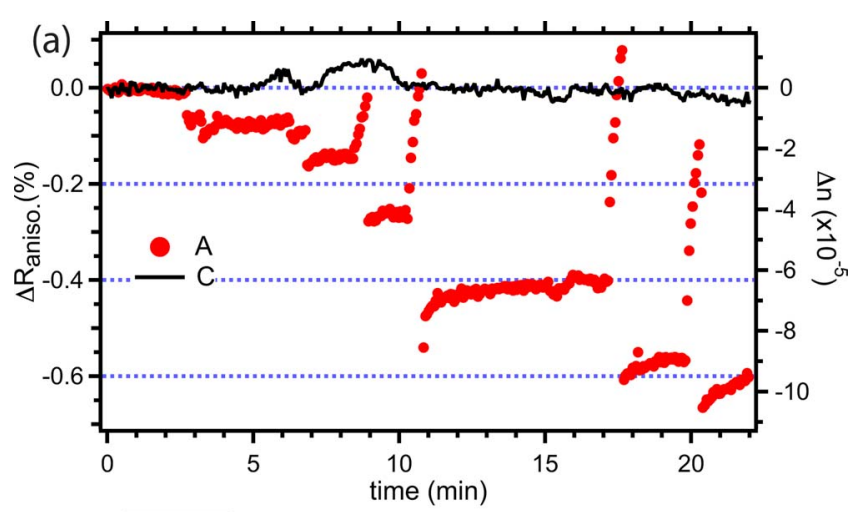

(b)

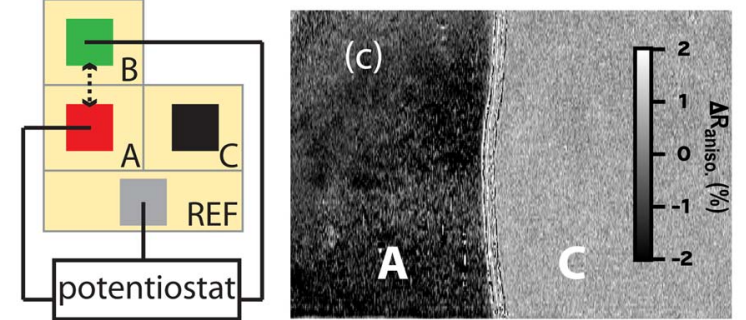

Fig. 3. (Color online) (a) Dynamical P-SPRI signal of a SAM-supported hemimembrane system. Dots, anisotropic response of electrode A. Line, response of electrode C (control). (b) Diagram of the biochip areas. (c) Image of the differential SPR anisotropy observed.

eraged SPR anisotropy of $<10^{-2} \%$ (or a bulk anisotropy of less than $10^{-5}$ ), while region A becomes anisotropic after the first cycle of the electric potential and gains anisotropy as more cycles are applied to the region. The sharp drops observed between steady state anisotropy levels are due to the electrical cycles themselves. As the potential applied changes polarity, the change in the orientation of the surface-bound molecules is believed to be maximal. At the end of the experiment, region A exhibits an averaged SPR anisotropy of $-0.6 \%$, yielding a bulk anisotropy of $\Delta n=-9 \times 10^{-5} \mathrm{RIU}$ or approximately $10^{-2}$ RIU for $1 \mathrm{~nm}$ of a dielectric material $(n=1.45)$ deposited.

The image of Fig. 3(c) is obtained by subtracting the differential SPR images at $t=1.5$ and $13.5 \mathrm{~min}$.
We can clearly observe that the region where electric potential cycles were applied gained a pattern, while the control region is still homogeneous at the end of the experiment. We believe that this patterning effect is responsible to some extent for the anisotropic signal observed.

A P-SPRI biosensor capable of resolving the dynamical anisotropy of biochemical films has been developed. The system is found to be limited by photon noise, while yielding a reading noise of less than $10^{-2} \%$, enabling the characterization of dynamical anisotropy of samples with a sensitivity on the order of $10^{-3}$ RIU on nanometric biological layers. A dynamical anisotropy measurement has been carried out on a biochemical film patterned by cycles of applied electrical potential, leading to a SPR signal of $-0.6 \%$ or $10^{-2} \mathrm{RIU}$ for $1 \mathrm{~nm}$ of a dielectric material $(n=1.45)$ deposited.

The authors acknowledge the support of the National French Research Agency (ANR) through the National Program for Nanosciences and Nanotechnologies (PNANO) of the European Network of Excellence Photonics4Life (FP7) and the help of A. Aide on the mechanical design.

\section{References}

1. J. Hottin, J. Moreau, G. Roger, J. Spadavecchia, M.-C. Millot, and M. Goosens, Plasmonics 2, 201 (2007).

2. B. P. Nelson, T. E. Grimsrud, M. R. Liles, R. M. Goodmab, and R. M. Corn, Anal. Chem. 73, 1 (2001).

3. D. R. Shankaran, K. V. Gobi, and M. Miura, Sens. Actuators B 121, 158 (2007).

4. M. Piliarik, H. Vaisocherov, and J. Homola, Biosens. Bioelectron. 20, 2104 (2005).

5. E. Kretschmann and H. Raether, Z. Naturforsch. A 23A, 2135 (1968).

6. Z. Salamon and G. Tollin, Biophys. J. 80, 1557 (2001).

7. M. Schubert, Thin Solid Films 313-314, 323 (1998).

8. R. Naumann, S. M. Schiller, F. Giess, B. Grohe, K. B. Hartman, I. Kärcher, I. Köper, J. Lübben, K. Vasilev, and W. Knoll, Langmuir 19, 5435 (2003).

9. P. Lecaruyer, E. Maillart, M. Canva, and J. Rolland, Appl. Opt. 45, 8419 (2006).

10. W. Boireau, S. Bombard, M. Sari, and D. Pompon, Biotechnol. Bioeng. 77, 225 (2002). 